\title{
Solvable graphs of finite groups
}

\author{
Parthajit Bhowal $^{1}$ (D), Deiborlang Nongsiang ${ }^{2}$ (D), Rajat Kanti Nath*1 (D) \\ ${ }^{1}$ Department of Mathematical Sciences, Tezpur University, Napaam-784028, Sonitpur, Assam, India \\ ${ }^{2}$ Department of Mathematics, Union Christian College, Umiam-793122, Meghalaya, India
}

\begin{abstract}
Let $G$ be a finite non-solvable group with solvable radical $\operatorname{Sol}(G)$. The solvable graph $\Gamma_{s}(G)$ of $G$ is a graph with vertex set $G \backslash \operatorname{Sol}(G)$ and two distinct vertices $u$ and $v$ are adjacent if and only if $\langle u, v\rangle$ is solvable. We show that $\Gamma_{s}(G)$ is not a star graph, a tree, an $n$-partite graph for any positive integer $n \geq 2$ and not a regular graph for any non-solvable finite group $G$. We compute the girth of $\Gamma_{s}(G)$ and derive a lower bound of the clique number of $\Gamma_{s}(G)$. We prove the non-existence of finite non-solvable groups whose solvable graphs are planar, toroidal, double-toroidal, triple-toroidal or projective. We conclude the paper by obtaining a relation between $\Gamma_{s}(G)$ and the solvability degree of $G$.
\end{abstract}

Mathematics Subject Classification (2010). 20D60, 05C25

Keywords. solvable graph, genus, solvability degree, finite group

\section{Introduction}

Let $G$ be a finite group and $u \in G$. The solvabilizer of $u$, denoted by $\operatorname{Sol}_{G}(u)$, is the set given by $\{v \in G:\langle u, v\rangle$ is solvable $\}$. Note that the centralizer $C_{G}(u):=\{v \in G: u v=v u\}$ is a subset of $\operatorname{Sol}_{G}(u)$ and hence the center $Z(G) \subseteq \operatorname{Sol}_{G}(u)$ for all $u \in G$. By [21, Proposition 2.13], $\left|C_{G}(u)\right|$ divides $\left|\operatorname{Sol}_{G}(u)\right|$ for all $u \in G$ though $\operatorname{Sol}_{G}(u)$ is not a subgroup of $G$ in general. A group $G$ is called a S-group if $\operatorname{Sol}_{G}(u)$ is a subgroup of $G$ for all $u \in G$. A finite group $G$ is a S-group if and only if it is solvable (see [21, Proposition 2.22]). Many other properties of $\operatorname{Sol}_{G}(u)$ can be found in [21]. We write $\operatorname{Sol}(G)=\{u \in G$ : $\langle u, v\rangle$ is solvable for all $v \in G\}$. It is easy to see that $\operatorname{Sol}(G)=\underset{u \in G}{\cap} \operatorname{Sol}_{G}(u)$. Also, $\operatorname{Sol}(G)$ is the solvable radical of $G$ (see [18]). The solvable graph of a finite non-solvable group $G$ is a simple undirected graph whose vertex set is $G \backslash \operatorname{Sol}(G)$, and two vertices $u$ and $v$ are adjacent if $\langle u, v\rangle$ is a solvable. We write $\Gamma_{s}(G)$ to denote this graph. It is worth mentioning that $\Gamma_{s}(G)$ is the complement of the non-solvable graph of $G$ considered in $[4,21]$ and extension of commuting and nilpotent graphs of finite groups that are studied extensively in $[1-3,5,6,9-11,13-16,25,26]$. It is worth mentioning that the study of commuting graphs of finite groups is originated from a question posed by Erdös [23].

In this paper, we show that $\Gamma_{s}(G)$ is not a star graph, a tree, an $n$-partite graph for any positive integer $n \geq 2$ and not a regular graph for any non-solvable finite group $G$. In Section 2, we also show that the girth of $\Gamma_{s}(G)$ is 3 and the clique number of $\Gamma_{s}(G)$ is

\footnotetext{
*Corresponding Author.

Email addresses: bhowal.parthajit8@gmail.com (P. Bhowal), ndeiborlang@yahoo.in (D. Nongsiang), rajatkantinath@yahoo.com (R.K. Nath)

Received: 05.06.2019; Accepted: 09.03.2020
} 
greater than or equal to 4 . In Section 3, we first show that for a given non-negative integer $k$, there are at the most finitely many finite non-solvable groups whose solvable graph have genus $k$. We also show that there is no finite non-solvable group, whose solvable graph is planar, toroidal, double-toroidal, triple-toroidal or projective. We conclude the paper by obtaining a relation between $\Gamma_{s}(G)$ and $P_{s}(G)$ in Section 4, where $P_{s}(G)$ is the probability that a randomly chosen pair of elements of $G$ generate a solvable group (see [20]).

The reader may refer to [27] and [28] for various standard graph theoretic terminologies. For any subset $X$ of the vertex set of a graph $\Gamma$, we write $\Gamma[X]$ to denote the induced subgraph of $\Gamma$ on $X$. The girth of $\Gamma$ is the minimum of the lengths of all cycles in $\Gamma$, and is denoted by girth $(\Gamma)$. We write $\omega(\Gamma)$ to denote the clique number of $\Gamma$ which is the least upper bound of the sizes of all the cliques of $\Gamma$. The smallest non-negative integer $k$ is called the genus of a graph $\Gamma$ if $\Gamma$ can be embedded on the surface obtained by attaching $k$ handles to a sphere. Let $\gamma(\Gamma)$ be the genus of $\Gamma$. Then, it is clear that $\gamma(\Gamma) \geq \gamma\left(\Gamma_{0}\right)$ for any subgraph $\Gamma_{0}$ of $\Gamma$. Let $K_{n}$ be the complete graph on $n$ vertices and $m K_{n}$ the disjoint union of $m$ copies of $K_{n}$. It was proved in [7, Corollary 1] that $\gamma(\Gamma) \geq \gamma\left(K_{m}\right)+\gamma\left(K_{n}\right)$ if $\Gamma$ has two disjoint subgraphs isomorphic to $K_{m}$ and $K_{n}$. Also, by [28, Theorem 6-38] we have

$$
\gamma\left(K_{n}\right)=\left\lceil\frac{(n-3)(n-4)}{12}\right\rceil \text { if } n \geq 3 .
$$

A graph $\Gamma$ is called planar, toroidal, double-toroidal and triple-toroidal if $\gamma(\Gamma)=0,1,2$ and 3 respectively.

Let $N_{k}$ be the connected sum of $k$ projective planes. A simple graph which can be embedded in $N_{k}$ but not in $N_{k-1}$, is called a graph of crosscap $k$. The notation $\bar{\gamma}(\Gamma)$ stand for the crosscap of a graph $\Gamma$. It is easy to see that $\bar{\gamma}(\Gamma) \geq \bar{\gamma}\left(\Gamma_{0}\right)$ for any subgraph $\Gamma_{0}$ of $\Gamma$. It was shown in [8] that

$$
\bar{\gamma}\left(K_{n}\right)= \begin{cases}\left\lceil\frac{1}{6}(n-3)(n-4)\right\rceil & \text { if } n \geq 3 \text { and } n \neq 7, \\ 3 & \text { if } n=7 .\end{cases}
$$

A graph $\Gamma$ is called a projective graph if $\bar{\gamma}(\Gamma)=1$. It is worth mentioning that $2 K_{5}$ is not projective graph (see [17]).

\section{Graph realization}

We begin with the following lemma.

Lemma 2.1. For every $u \in G \backslash \operatorname{Sol}(G)$ we have

$$
\operatorname{deg}(u)=\left|\operatorname{Sol}_{G}(u)\right|-|\operatorname{Sol}(G)|-1 .
$$

Proof. Note that $\operatorname{deg}(u)$ represents the number of vertices from $G \backslash \operatorname{Sol}(G)$ which are adjacent to $u$. Since $u \in \operatorname{Sol}_{G}(u)$, therefore $\left|\operatorname{Sol}_{G}(u)\right|-1$ represents the number of vertices which are adjacent to $u$. Since we are excluding $\operatorname{Sol}(G)$ from the vertex set therefore $\operatorname{deg}(u)=\left|\operatorname{Sol}_{G}(u)\right|-|\operatorname{Sol}(G)|-1$.

Proposition 2.2. $\Gamma_{s}(G)$ is not a star.

Proof. Suppose for a contradiction $\Gamma_{s}(G)$ is a star. Let $|G|-|\operatorname{Sol}(G)|=n$. Then there exists $u \in G \backslash \operatorname{Sol}(G) \operatorname{such}$ that $\operatorname{deg}(u)=n-1$. Therefore, by Lemma 2.1, $\left|\operatorname{Sol}_{G}(u)\right|=|G|$. This gives $u \in \operatorname{Sol}(G)$, a contradiction. Hence, the result follows.

Proposition 2.3. $\Gamma_{s}(G)$ is not complete bipartite.

Proof. Let $\Gamma_{s}(G)$ be complete bipartite. Suppose that $A_{1}$ and $A_{2}$ are parts of the bipartition. Then, by Proposition 2.2, $\left|A_{1}\right| \geq 2$ and $\left|A_{2}\right| \geq 2$. Let $u \in A_{1}, v \in A_{2}$. If $|\langle u, v\rangle \operatorname{Sol}(G) \backslash \operatorname{Sol}(G)|>2$, then there exists $y \in\langle u, v\rangle \operatorname{Sol}(G) \backslash \operatorname{Sol}(G)$ with $u \neq y \neq v$ such that $\langle u, y\rangle$ and $\langle v, y\rangle$ are both solvable. But then $y \notin A_{1}$ and $y \notin A_{2}$, a contradiction. 
It follows that $|\langle u, v\rangle \operatorname{Sol}(G) \backslash \operatorname{Sol}(G)|=2$. In particular, $\operatorname{Sol}(G)=1$ and $\langle u, v\rangle$ is cyclic of order 3 or $|\operatorname{Sol}(G)|=2$ and $v=u z$ for $z$ an involution in $\operatorname{Sol}(G)$. Now the neighbours of $u \in A_{1}$ is just $u^{2} \in A_{2}$ or $u z$ in the respective cases. Hence $\left|A_{2}\right|=\left|A_{1}\right|=1$, a contradiction. Hence, the result follows.

Following similar arguments as in the proof of Proposition 2.3 we get the following result.

Proposition 2.4. $\Gamma_{s}(G)$ is not complete n-partite.

Proposition 2.5. For any finite non-solvable group $G, \Gamma_{s}(G)$ has no isolated vertex.

Proof. Suppose $x$ is an isolated vertex of $\Gamma_{s}(G)$. Then $|\operatorname{Sol}(G)|=1$; otherwise $x$ is adjacent to $x z$ for any $z \in \operatorname{Sol}(G) \backslash\{1\}$. Thus it follows that $o(x)=2$; otherwise $x$ is adjacent to $x^{2}$. Let $y \in G$. Then $\left\langle x, x^{y}\right\rangle$ is dihedral and so $x=x^{y}$ as $x$ is isolated. Hence $x \in Z(G)$ and so $x \in Z(G) \leq \operatorname{Sol}(G)$, a contradiction. Hence, $\Gamma_{s}(G)$ has no isolated vertex.

The following lemma is useful in proving the next two results as well as some results in subsequent sections.

Lemma 2.6. Let $G$ be a finite non-solvable group. Then there exist $x \in G$ such that $x, x^{2} \notin \operatorname{Sol}(G)$.

Proof. Suppose that for all $x \in G$, we have $x^{2} \in \operatorname{Sol}(G)$. Therefore, $G / \operatorname{Sol}(G)$ is elementary abelian and hence solvable. Also, $\operatorname{Sol}(G)$ is solvable. It follows that $G$ is solvable, a contradiction. Hence, the result follows.

Theorem 2.7. Let $G$ be a finite non-solvable group. Then $\operatorname{girth}\left(\Gamma_{s}(G)\right)=3$.

Proof. Suppose for a contradiction that $\Gamma_{s}(G)$ has no 3-cycle. Let $x \in G$ such that $x, x^{2} \notin \operatorname{Sol}(G)$ (by Lemma 2.6). Suppose $|\operatorname{Sol}(G)| \geq 2$. Let $z \in \operatorname{Sol}(G), z \neq 1$, then $x, x^{2}$ and $x z$ form a 3 -cycle, which is a contradiction. Thus $|\operatorname{Sol}(G)|=1$. In this case, every element of $G$ has order 2 or 3 ; otherwise, $\left\{x, x^{2}, x^{3}\right\}$ forms a 3-cycle in $\Gamma_{s}(G)$ for all $x \in G$ with $o(x)>3$. Therefore, $|G|=2^{m} 3^{n}$ for some non-negative integers $m$ and $n$. By Burnside's Theorem, it follows that $G$ is solvable; a contradiction. Hence, $\operatorname{girth}\left(\Gamma_{s}(G)\right)=3$.

Theorem 2.8. Let $G$ be a finite non-solvable group. Then $\omega\left(\Gamma_{s}(G)\right) \geq 4$.

Proof. Suppose for a contradiction that $G$ is a finite non-solvable group with $\omega\left(\Gamma_{s}(G)\right) \leq$ 3. Let $x \in G \backslash \operatorname{Sol}(G)$ such that $x^{2} \notin \operatorname{Sol}(G)$ according to Lemma 2.6. Suppose $|\operatorname{Sol}(G)| \geq$ 2. Let $z \in \operatorname{Sol}(G), z \neq 1$, then $\left\{x, x^{2}, x z, x^{2} z\right\}$ is a clique which is a contradiction. Thus $|\operatorname{Sol}(G)|=1$. In this case every element of $G \backslash \operatorname{Sol}(G)$ has order 2,3 or 4 otherwise $\left\{x, x^{2}, x^{3}, x^{4}\right\}$ is a clique with $o(x)>4$, which is a contradiction. Therefore $|G|=2^{m} 3^{n}$ where $m, n$ are non-negative integers. Again, by Burnside's Theorem, it follows that $G$ is solvable; a contradiction. This completes the proof.

As a consequence of Theorem 2.7 and Theorem 2.8 we have the following corollary.

Corollary 2.9. The solvable graph of a finite non-solvable group is not a tree.

We conclude this section with the following result.

Proposition 2.10. $\Gamma_{s}(G)$ is not regular.

Proof. Follows from [21, Corollary 3.17], noting the fact that a graph is regular if and only if its complement is regular. 


\section{Genus and diameter}

We begin this section with the following useful lemma.

Lemma 3.1. Let $G$ be a finite group and $H$ a solvable subgroup of $G$. Then $\langle H, \operatorname{Sol}(G)\rangle$ is a solvable subgroup of $G$.

Proposition 3.2. Let $G$ be a finite non-solvable group such that $\gamma\left(\Gamma_{s}(G)\right)=m$.

(a) If $S$ is a nonempty subset of $G \backslash \operatorname{Sol}(G)$ such that $\langle x, y\rangle$ is solvable for all $x, y \in S$, then $|S| \leq\left|\frac{7+\sqrt{1+48 m}}{2}\right|$.

(b) $|\operatorname{Sol}(G)| \leq \frac{1}{t-1}\left\lfloor\frac{7+\sqrt{1+48 m}}{2}\right\rfloor$, where $t=\max \{o(x \operatorname{Sol}(G)) \mid x \operatorname{Sol}(G) \in G / \operatorname{Sol}(G)\}$.

(c) If $H$ is a solvable subgroup of $G$, then $|H| \leq\left\lfloor\frac{7+\sqrt{1+48 m}}{2}\right\rfloor+|H \cap \operatorname{Sol}(G)|$.

Proof. We have $\Gamma_{s}(G)[S] \cong K_{|S|}$ and $\gamma\left(K_{|S|}\right)=\gamma\left(\Gamma_{s}(G)[S]\right) \leq \gamma\left(\Gamma_{s}(G)\right)$. Therefore, if $m=0$ then $\gamma\left(K_{|S|}\right)=0$. This gives $|S| \leq 4$, otherwise $K_{|S|}$ will have a subgraph $K_{5}$ having genus 1. If $m>0$ then, by Heawood's formula [27, Theorem 6.3.25], we have

$$
|S|=\omega\left(\Gamma_{s}(G)[S]\right) \leq \omega\left(\Gamma_{s}(G)\right) \leq \chi\left(\Gamma_{s}(G)\right) \leq\left\lfloor\frac{7+\sqrt{1+48 m}}{2}\right\rfloor
$$

where $\chi\left(\Gamma_{s}(G)\right)$ is the chromatic number of $\Gamma_{s}(G)$. Hence part (a) follows.

Part (b) follows from Lemma 3.1 and part (a) considering $S=\bigsqcup_{i=1}^{t-1} y^{i} \operatorname{Sol}(G)$, where $y \in G \backslash \operatorname{Sol}(G)$ such that $o(y \operatorname{Sol}(G))=t$.

Part (c) follows from part (a) noting that $H=(H \backslash \operatorname{Sol}(G)) \cup(H \cap \operatorname{Sol}(G))$.

Theorem 3.3. Let $G$ be a finite non-solvable group. Then $|G|$ is bounded above by a function of $\gamma\left(\Gamma_{s}(G)\right)$.

Proof. Let $\gamma\left(\Gamma_{s}(G)\right)=m$ and $h_{m}=\left\lfloor\frac{7+\sqrt{1+48 m}}{2}\right\rfloor$. By Lemma 3.1, we have $\Gamma_{s}(G)[x \operatorname{Sol}(G)]$ $\cong K_{|\operatorname{Sol}(G)|}$, where $x \in G \backslash \operatorname{Sol}(G)$. Therefore by Proposition 3.2(a), $|\operatorname{Sol}(G)| \leq h_{m}$.

Let $P$ be a Sylow $p$-subgroup of $G$ for any prime $p$ dividing $|G|$ having order $p^{n}$ for some positive integer $n$. Then $P$ is a solvable. Therefore, by Proposition 3.2(c), we have $|P| \leq h_{m}+|\operatorname{Sol}(G)| \leq 2 h_{m}$. Hence, $|G|<\left(2 h_{m}\right)^{h_{m}}$ noting that the number of primes less than $2 h_{m}$ is at most $h_{m}$. This completes the proof.

As an immediate consequence of Theorem 3.3 we have the following corollary.

Corollary 3.4. Let $n$ be a non-negative integer. Then there are at the most finitely many finite non-solvable groups $G$ such that $\gamma\left(\Gamma_{s}(G)\right)=n$.

The following two lemmas are essential in proving the main results of this section.

Lemma 3.5. [24, Lemma 3.4] Let $G$ be a finite group.

(a) If $|G|=7 m$ and the Sylow 7-subgroup is normal in $G$, then $G$ has an abelian subgroup of order at least 14 or $|G| \leq 42$.

(b) If $|G|=9 m$, where $3 \nmid m$ and the Sylow 3 -subgroup is normal in $G$, then $G$ has an abelian subgroup of order at least 18 or $|G| \leq 72$.

Lemma 3.6. If $G$ is a non-solvable group of order not exceeding 120 then $\Gamma_{s}(G)$ has a subgraph isomorphic to $K_{11}$ and $\gamma\left(\Gamma_{s}(G)\right) \geq 5$.

Proof. If $G$ is a non-solvable group and $|G| \leq 120$ then $G$ is isomorphic to $A_{5}, A_{5} \times \mathbb{Z}_{2}, S_{5}$ or $S L(2,5)$. Note that $\left|\operatorname{Sol}\left(A_{5}\right)\right|=\left|\operatorname{Sol}\left(S_{5}\right)\right|=1$ and $\left|\operatorname{Sol}\left(A_{5} \times \mathbb{Z}_{2}\right)\right|=|\operatorname{Sol}(S L(2,5))|=2$. Also, $A_{5}$ has a solvable subgroup of order 12 and $S_{5}, A_{5} \times \mathbb{Z}_{2}, S L(2,5)$ have solvable subgroups of order 24 . It follows that $\Gamma_{s}(G)$ has a subgraph isomorphic to $K_{11}$. Therefore, by $(1.1), \gamma\left(\Gamma_{s}(G)\right) \geq \gamma\left(K_{11}\right)=5$. 
Theorem 3.7. The solvable graph of a finite non-solvable group is neither planar, toroidal, double-toroidal nor triple-toroidal.

Proof. Let $G$ be a finite non-solvable group. Note that it is enough to show $\gamma\left(\Gamma_{s}(G)\right) \geq 4$ to complete the proof. Suppose that $\gamma\left(\Gamma_{s}(G)\right) \leq 3$. Let $x \in G \backslash \operatorname{Sol}(G)$ such that $x^{2} \notin \operatorname{Sol}(G)$. Such element exists by Lemma 2.6. Since any two elements of the set $A=$ $x \operatorname{Sol}(G) \cup x^{2} \operatorname{Sol}(G)$ generate a solvable group, by Proposition 3.2(a), we have $2|\operatorname{Sol}(G)|=$ $|A| \leq\left\lfloor\frac{7+\sqrt{1+48 \cdot 3}}{2}\right\rfloor=9$. Thus $|\operatorname{Sol}(G)| \leq 4$. Let $p$ be a prime divisor of $|G|$ and $P$ is a Sylow $p$-subgroup of $G$. Since $P$ is solvable, by Proposition 3.2(c), we get $|P| \leq 9+|P \cap \operatorname{Sol}(G)| \leq$ 13. If $|P|=11$ or 13 then $|P \cap \operatorname{Sol}(G)|=1$. Therefore, $\Gamma_{s}(G)[P \backslash \operatorname{Sol}(G)] \cong K_{10}$ or $K_{12}$. Using (1.1), we get $\gamma\left(\Gamma_{s}(G)[P \backslash \operatorname{Sol}(G)]\right)=4$ or 6 . Therefore, $\gamma\left(\Gamma_{s}(G)\right) \geq$ $\gamma\left(\Gamma_{s}(G)[P \backslash \operatorname{Sol}(G)]\right) \geq 4$, a contradiction. Thus $|P| \leq 9$ and hence $p \leq 7$. This shows that $|G|$ divides $2^{3} .3^{2} .5 .7$.

We consider the following cases.

Case 1. $|\operatorname{Sol}(G)|=4$.

If $H$ is a Sylow $p$-subgroup of $G$ where $p=5$ or 7 then $\langle H, \operatorname{Sol}(G)\rangle$ is solvable since $H$ is solvable (by Lemma 3.1). We have $|H \cap \operatorname{Sol}(G)|=1$ and $\mid\langle H$, $\operatorname{Sol}(G)\rangle \mid=20,28$ according as $p=5,7$ respectively. Therefore $\Gamma_{s}(G)[\langle H, \operatorname{Sol}(G)\rangle \backslash \operatorname{Sol}(G)] \cong K_{16}$ or $K_{24}$. By (1.1) we get $\gamma\left(\Gamma_{s}(G)\right) \geq \gamma\left(\Gamma_{s}(G)[\langle H, \operatorname{Sol}(G)\rangle \backslash \operatorname{Sol}(G)]\right) \geq 13$, which is a contradiction.

Thus $|G|$ is a divisor of 72 . Therefore, by Lemma 3.6 we have $\gamma\left(\Gamma_{s}(G)\right) \geq 5$, a contradiction.

Case 2. $|\operatorname{Sol}(G)|=3$.

If $H$ is a Sylow $p$-subgroup of $G$ where $p=5$ or 7 then $\langle H, \operatorname{Sol}(G)\rangle$ is solvable. We have $|H \cap \operatorname{Sol}(G)|=1$ and $|\langle H, \operatorname{Sol}(G)\rangle|=15,21$ according as $p=5,7$ respectively. Therefore $\Gamma_{s}(G)[\langle H, \operatorname{Sol}(G)\rangle \backslash \operatorname{Sol}(G)] \cong K_{12}$ or $K_{18}$. By (1.1) we get $\gamma\left(\Gamma_{s}(G)\right) \geq$ $\gamma\left(\Gamma_{s}(G)[\langle H, \operatorname{Sol}(G)\rangle \backslash \operatorname{Sol}(G)]\right) \geq 6$, which is a contradiction.

Thus $|G|$ is a divisor of 72 . Therefore, by Lemma 3.6 we have $\gamma\left(\Gamma_{s}(G)\right) \geq 5$, a contradiction.

Case 3. $|\operatorname{Sol}(G)|=2$.

If $H$ is a Sylow 7-subgroup of $G$ then $\langle H, \operatorname{Sol}(G)\rangle$ is solvable. We have $|H \cap \operatorname{Sol}(G)|=1$ and $|\langle H, \operatorname{Sol}(G)\rangle|=14$. So, $\Gamma_{s}(G)[\langle H, \operatorname{Sol}(G)\rangle \backslash \operatorname{Sol}(G)] \cong K_{12}$. By $(1.1)$ we get $\gamma\left(\Gamma_{s}(G)\right) \geq$ $\gamma\left(\Gamma_{s}(G)[\langle H, \operatorname{Sol}(G)\rangle \backslash \operatorname{Sol}(G)]\right) \geq 6$, which is a contradiction. Let $K$ be a Sylow 3-subgroup of $G$. If $|K|=9$ then $\langle K, \operatorname{Sol}(G)\rangle$ is solvable since $K$ is solvable (by Lemma 3.1). We have $|K \cap \operatorname{Sol}(G)|=1$ and $|\langle K, \operatorname{Sol}(G)\rangle|=18$. So, $\Gamma_{s}(G)[\langle K, \operatorname{Sol}(G)\rangle \backslash \operatorname{Sol}(G)] \cong K_{16}$. By (1.1) we get $\gamma\left(\Gamma_{s}(G)\right) \geq \gamma\left(\Gamma_{s}(G)[\langle K, \operatorname{Sol}(G)\rangle \backslash \operatorname{Sol}(G)]\right)=13$, which is a contradiction.

Thus $|G|$ is a divisor of 120 . Therefore, by Lemma 3.6 we have $\gamma\left(\Gamma_{s}(G)\right) \geq 5$, a contradiction.

Case 4. $|\operatorname{Sol}(G)|=1$.

In this case, first we shall show that $7 \nmid|G|$. On the contrary, assume that 7||$G \mid$. Let $n$ be the number of Sylow 7-subgroups of $G$. Then $n \mid 2^{3} .3^{2} .5$ and $n \equiv 1 \bmod 7$. If $n \neq 1$ then $n \geq 8$. Let $H_{1}, \ldots, H_{8}$ be the eight distinct Sylow 7-subgroups of $G$. Then the induced subgraphs $\Gamma_{S}(G)\left[H_{i} \backslash \operatorname{Sol}(G)\right]$ for each $1 \leq i \leq 8$ contribute $\gamma\left(\Gamma_{S}(G)\left[H_{i} \backslash \operatorname{Sol}(G)\right]\right)=1$ to the genus of $\Gamma_{S}(G)$. Thus

$$
\gamma\left(\Gamma_{S}(G)\right) \geq \sum_{i=1}^{8} \gamma\left(\Gamma_{S}(G)\left[H_{i} \backslash \operatorname{Sol}(G)\right]\right)=8,
$$

a contradiction. Therefore, Sylow 7-subgroup of $G$ is unique and hence normal. Since we have started with a non-solvable group, by Lemma 3.5, it follows that $G$ has an abelian subgroup of order at least 14 . Therefore, by (1.1) we have $\gamma\left(\Gamma_{S}(G)\right) \geq \gamma\left(K_{13}\right)=8$, a contradiction. Hence, $|G|$ is a divisor of $2^{3} .3^{2} .5$.

Now, we shall show that $9 \nmid|G|$. Assume that, on the contrary, 9||$G \mid$. If Sylow 3subgroup of $G$ is not normal in $G$, then the number of Sylow 3 -subgroups is greater than 
or equal to 4. Let $H_{1}, H_{2}, H_{3}$ be the three Sylow 3-subgroups of $G$. Then the induced subgraph $\Gamma_{S}(G)\left[H_{1} \backslash \operatorname{Sol}(G)\right] \cong K_{8}$ and so it contributes $\gamma\left(\Gamma_{S}(G)\left[H_{1} \backslash \operatorname{Sol}(G)\right]\right)=2$ to the genus of $\Gamma_{S}(G)$. If $\left|H_{1} \cap H_{2}\right|=1$, then the induced subgraph $\Gamma_{S}(G)\left[H_{2} \backslash \operatorname{Sol}(G)\right] \cong K_{8}$ and so it contributes +2 to the genus $\Gamma_{S}(G)$. Thus

$$
\gamma\left(\Gamma_{S}(G)\right) \geq \gamma\left(\Gamma_{S}(G)\left[\left(H_{1} \cup H_{2}\right) \backslash \operatorname{Sol}(G)\right]\right)=4
$$

which is a contradiction. So assume that $\left|H_{1} \cap H_{2}\right|=3$. Similarly $\left|H_{1} \cap H_{3}\right|=3$ and $\left|H_{2} \cap H_{3}\right|=3$. Let $M=H_{2} \backslash H_{1}$. Then $|M|=6$. Also note that if $L=H_{1} \cup H_{2}$ and $K=H_{3} \backslash L$, then $|K| \geq 4$. Also $H_{1} \cap M=H_{1} \cap K=M \cap K=\emptyset$.

If $|K| \geq 5$ then $H_{1}$ contribute +2 to genus of $\Gamma_{S}(G), M$ and $K$ each contribute +1 to genus of $\Gamma_{S}(G)$. Hence genus of $\Gamma_{S}(G)$ is greater than or equal to 4 , a contradiction.

Assume that $|K|=4$. In this case $\left|M \cap H_{3}\right|=2$. Let $x \in M \cap H_{3}$. Then $H_{1}$ contribute +2 to genus of $\Gamma_{S}(G), M \backslash\{x\}$ and $K \cup\{x\}$ each contribute +1 to genus of $\Gamma_{S}(G)$. Hence genus of $\Gamma_{S}(G)$ is greater than or equal to 4 , a contradiction.

These show that Sylow 3-subgroup of $G$ is unique and hence normal in $G$. Therefore, by Lemma 3.5 and Lemma 3.6, $G$ has an abelian subgroup $A$ of order at least 18. Hence,

$$
\gamma\left(\Gamma_{S}(G)\right) \geq \gamma\left(\Gamma_{S}(G)[A \backslash \operatorname{Sol}(G)]\right) \geq \gamma\left(K_{17}\right)=16
$$

which is a contradiction.

It follows that $9 \nmid|G|$ and $|G|$ is a divisor of 120 . Therefore, by Lemma 3.6 we get $\gamma\left(\Gamma_{S}(G)\right) \geq 5$, a contradiction. Hence, $\gamma\left(\Gamma_{s}(G)\right) \geq 4$ and the result follows.

The above theorem gives that $\gamma\left(\Gamma_{s}(G)\right) \geq 4$. Usually, genera of solvable graphs of finite non-solvable groups are very large. For example, if $G$ is the smallest non-solvable group $A_{5}$ then $\Gamma_{s}(G)$ has 59 vertices and 571 edges. Also $\gamma\left(\Gamma_{s}(G)\right) \geq 571 / 6-59 / 2+1=68$ (follows from [28, Corollary 6-14]). The following theorem shows that the crosscap number of the solvable graph of a finite non-solvable group is greater than 1 .

Proposition 3.8. The solvable graph of a finite non-solvable group is not projective.

Proof. Suppose $G$ is a finite non-solvable group whose solvable graph is projective. Note that if $\Gamma_{s}(G)$ has a subgraph isomorphic to $K_{n}$ then, by (1.2), we must have $n \leq 6$. Let $x \in G$, such that $x, x^{2} \notin \operatorname{Sol}(G)$. Then

$$
\Gamma_{s}(G)\left[x \operatorname{Sol}(G) \cup x^{2} \operatorname{Sol}(G)\right] \cong K_{2|\operatorname{Sol}(G)|} .
$$

Therefore, $2|\operatorname{Sol}(G)| \leq 6$ and hence $|\operatorname{Sol}(G)| \leq 3$.

Let $p|| G \mid$ be a prime and $P$ be a Sylow $p$-subgroup of $G$. Then $\Gamma_{s}(G)[P \backslash \operatorname{Sol}(G)] \cong$ $K_{|P \backslash \operatorname{Sol}(G)|}$ since $P$ is solvable. Therefore, $|P \backslash \operatorname{Sol}(G)|=|P|-|P \cap \operatorname{Sol}(G)| \leq 6$ and hence $|P| \leq 9$. This shows that $|G|$ is a divisor of $2^{3} .3^{2} .5 .7$.

If 7||$G \mid$ then Sylow 7-subgroup of $G$ is unique and hence normal in $G$; otherwise, let $H$ and $K$ be two Sylow 7-subgroups of $G$. Then $|H \cap K|=|H \cap \operatorname{Sol}(G)|=|K \cap \operatorname{Sol}(G)|=1$. Therefore, $\Gamma_{s}(G)[(H \cup K) \backslash \operatorname{Sol}(G)]$ has a subgraph isomorphic to $2 K_{6}$. Hence, $\Gamma_{s}(G)$ has a subgraph isomorphic to $2 K_{5}$, which is a contradiction. Similarly, if 9||$G \mid$, then the Sylow 3 -subgroup of $G$ is normal in $G$. Therefore, by Lemma 3.5, it follows that $|G| \leq 72$ or $|G|$ is a divisor of $2^{3} .3 .5$. In the both cases, by Lemma 3.6, $\Gamma_{s}(G)$ has complete subgraphs isomorphic to $K_{11}$, which is a contradiction. This completes the proof.

We conclude this section, by an observation and a couple of problems regarding the diameter and connectedness of $\Gamma_{s}(G)$. Using the following programme in GAP[29], we see that the solvable graph of the groups $A_{5}, S_{5}, A_{5} \times \mathbb{Z}_{2}, S L(2,5), P S L(3,2)$ and $G L(2,4)$ are connected with diameter 2 . The solvable graphs of $S_{6}$ and $A_{6}$ are connected with diameters greater than 2 . 


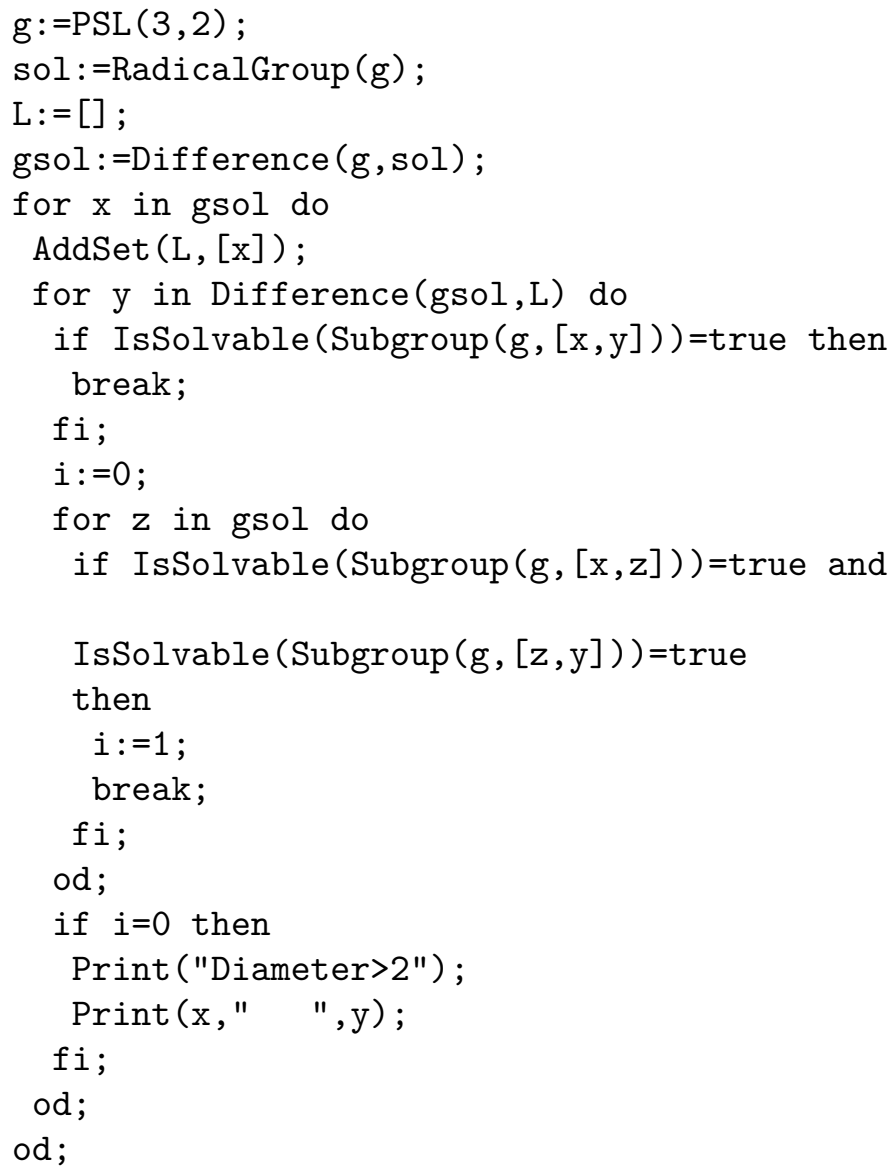

In this connection, we have the following problems.

Problem 3.1. Is $\Gamma_{s}(G)$ connected for any finite non-solvable group $G$ ?

Problem 3.2. Is there any finite bound for the diameter of $\Gamma_{s}(G)$ when $\Gamma_{s}(G)$ is connected?

\section{Relations with solvability degree}

The solvability degree of a finite group $G$ is defined by the following ratio

$$
P_{s}(G):=\frac{\mid\{(u, v) \in G \times G:\langle u, v\rangle \text { is solvable }\} \mid}{|G|^{2}} .
$$

Using the solvability criterion (see [12, Section 1]),

"A finite group is solvable if and only if every pair of its elements generates a solvable group"

for finite groups we have $G$ is solvable if and only if its solvability degree is 1 . It was shown in [20, Theorem A] that $P_{s}(G) \leq \frac{11}{30}$ for any finite non-solvable group $G$. In this section, we study a few properties of $P_{s}(G)$ and derive a connection between $P_{s}(G)$ and $\Gamma_{s}(G)$ for finite non-solvable groups $G$. We begin with the following lemma.

Lemma 4.1. Let $G$ be a finite group. Then $P_{s}(G)=\frac{1}{|G|^{2}} \sum_{u \in G}\left|\operatorname{Sol}_{G}(u)\right|$.

Proof. Let $\mathcal{S}=\{(u, v) \in G \times G:\langle u, v\rangle$ is solvable $\}$. Then

$$
\mathcal{S}=\cup_{u \in G}(\{u\} \times\{v \in G:\langle u, v\rangle \text { is solvable }\})=\cup_{u \in G}\left(\{u\} \times \operatorname{Sol}_{G}(u)\right) .
$$

Therefore, $|\mathcal{S}|=\sum_{u \in G}\left|\operatorname{Sol}_{G}(u)\right|$. Hence, the result follows. 
Corollary 4.2. $|G| P_{s}(G)$ is an integer for any finite group $G$.

Proof. By Proposition 2.16 of [21] we have that $|G|$ divides $\sum_{u \in G}\left|\operatorname{Sol}_{G}(u)\right|$. Hence, the result follows from Lemma 4.1.

We have the following lower bound for $P_{s}(G)$.

Theorem 4.3. For any finite group $G$,

$$
P_{s}(G) \geq \frac{|\operatorname{Sol}(G)|}{|G|}+\frac{2(|G|-|\operatorname{Sol}(G)|)}{|G|^{2}} .
$$

Proof. By Lemma 4.1, we have

$$
\begin{aligned}
|G|^{2} P_{s}(G) & =\sum_{u \in \operatorname{Sol}(G)}\left|\operatorname{Sol}_{G}(u)\right|+\sum_{u \in G \backslash \operatorname{Sol}(G)}\left|\operatorname{Sol}_{G}(u)\right| \\
& =|G||\operatorname{Sol}(G)|+\sum_{u \in G \backslash \operatorname{Sol}(G)}\left|\operatorname{Sol}_{G}(u)\right| .
\end{aligned}
$$

By Proposition 2.13 of [21], $\left|C_{G}(u)\right|$ is a divisor of $\left|\operatorname{Sol}_{G}(u)\right|$ for all $u \in G$ where $C_{G}(u)=$ $\{v \in G: u v=v u\}$, the centralizer of $u \in G$. Since $\left|C_{G}(u)\right| \geq 2$ for all $u \in G$ we have $\left|\operatorname{Sol}_{G}(u)\right| \geq 2$ for all $u \in G$. Therefore

$$
\sum_{u \in G \backslash \operatorname{Sol}(G)}\left|\operatorname{Sol}_{G}(u)\right| \geq 2(|G|-|\operatorname{Sol}(G)|) .
$$

Hence, the result follows from (4.1).

The following theorem shows that $P_{s}(G)>\operatorname{Pr}(G)$ for any finite non-solvable group where $\operatorname{Pr}(G)$ is the commuting probability of $G$ (see [19]).

Theorem 4.4. Let $G$ be a finite group. Then $P_{s}(G) \geq \operatorname{Pr}(G)$ with equality if and only if $G$ is a solvable group.

Proof. The result follows from Lemma 4.1 and the fact that $\operatorname{Pr}(G)=\frac{1}{|G|^{2}} \sum_{u \in G}\left|C_{G}(u)\right|$ noting that $C_{G}(u) \subseteq \operatorname{Sol}_{G}(u)$ and so $\left|\operatorname{Sol}_{G}(u)\right| \geq\left|C_{G}(u)\right|$ for all $u \in G$.

The equality holds if and only if $C_{G}(u)=\operatorname{Sol}_{G}(u)$ for all $u \in G$, that is $\operatorname{Sol}_{G}(u)$ is a subgroup of $G$ for all $u \in G$. Hence, by Proposition 2.22 of [21], the equality holds if and only if $G$ is solvable.

Let $\left|E\left(\Gamma_{s}(G)\right)\right|$ be the number of edges of the non-solvable graph $\Gamma_{s}(G)$ of $G$. The following theorem gives a relation between $P_{s}(G)$ and $\left|E\left(\Gamma_{s}(G)\right)\right|$.

Theorem 4.5. Let $G$ be a finite non-solvable group. Then

$$
2\left|E\left(\Gamma_{s}(G)\right)\right|=|G|^{2} P_{s}(G)+|\operatorname{Sol}(G)|^{2}+|\operatorname{Sol}(G)|-|G|(2|\operatorname{Sol}(G)|+1) .
$$

Proof. We have

$2\left|E\left(\Gamma_{s}(G)\right)\right|=\mid\{(x, y) \in(G \backslash \operatorname{Sol}(G)) \times(G \backslash \operatorname{Sol}(G)):\langle x, y\rangle$ is solvable $\}|-| G|+| \operatorname{Sol}(G) \mid$.

Also

$$
\begin{aligned}
\mathcal{S}= & \{(x, y) \in G \times G:\langle x, y\rangle \text { is solvable }\} \\
= & \operatorname{Sol}(G) \times \operatorname{Sol}(G) \quad \sqcup \quad \operatorname{Sol}(G) \times(G \backslash \operatorname{Sol}(G)) \quad \sqcup \quad(G \backslash \operatorname{Sol}(G)) \times \operatorname{Sol}(G) \\
& \sqcup \quad\{(x, y) \in(G \backslash \operatorname{Sol}(G)) \times(G \backslash \operatorname{Sol}(G)):\langle x, y\rangle \text { is solvable }\} .
\end{aligned}
$$

Therefore

$$
\begin{aligned}
|\mathcal{S}| & =|\operatorname{Sol}(G)|^{2}+2|\operatorname{Sol}(G)|(|G|-|\operatorname{Sol}(G)|)+2\left|E\left(\Gamma_{s}(G)\right)\right|+|G|-|\operatorname{Sol}(G)| \\
\Longrightarrow|G|^{2} P_{s}(G) & =|G|(2|\operatorname{Sol}(G)|+1)-|\operatorname{Sol}(G)|^{2}-|\operatorname{Sol}(G)|+2\left|E\left(\Gamma_{s}(G)\right)\right| .
\end{aligned}
$$

Hence, the result follows. 
We conclude this paper noting that lower bounds for $\left|E\left(\Gamma_{s}(G)\right)\right|$ can be obtained from Theorem 4.5 using the lower bounds given in Theorem 4.3, Theorem 4.4 and the lower bounds for $\operatorname{Pr}(G)$ obtained in [22].

Acknowledgment. The authors would like to thank the referee for his/her valuable comments and suggestions. The first author is thankful to Council of Scientific and Industrial Research for the fellowship (File No. 09/796(0094)/2019-EMR-I).

\section{References}

[1] A. Abdollahi, M. Zarrin, Non-nilpotent graph of a group, Comm. Algebra, 38 (12), 4390-4403, 2010.

[2] A. Abdollahi, S. Akbari and H.R. Maimani, Non-commuting graph of a group, J. Algebra, 298 (2), 468-492, 2006.

[3] M. Afkhami, D.G.M. Farrokhi and K. Khashyarmanesh, Planar, toroidal, and projective commuting and non-commuting graphs, Comm. Algebra, 43 (7), 2964-2970, 2015.

[4] B. Akbari, More on the Non-Solvable Graphs and Solvabilizers, arXiv:1806.01012v1, 2018.

[5] S. Akbari, A. Mohammadian, H. Radjavi and P. Raja, On the diameters of commuting graphs, Linear Algebra Appl. 418 (1), 161-176, 2006.

[6] C. Bates, D. Bundy, S. Hart and P. Rowley, A Note on Commuting Graphs for Symmetric Groups, Electron. J. Combin. 16 (1), R6:1-13, 2009.

[7] J. Battle, F. Harary, Y. Kodama and J.W.T. Youngs, Additivity of the genus of a graph, Bull. Amer. Math. Soc. 68 (6), 565-568, 1962.

[8] A. Bouchet, Orientable and nonorientable genus of the complete bipartite graph, J. Combin. Theory Ser. B, 24 (1), 24-33, 1978.

[9] M.R. Darafsheh, H. Bigdely, A. Bahrami and M.D. Monfared, Some results on noncommuting graph of a finite group, Ital. J. Pure Appl. Math. 268, 371-387, 2010.

[10] A.K. Das and D. Nongsiang, On the genus of the nilpotent graphs of finite groups, Comm. Algebra 43 (12), 5282-5290, 2015.

[11] A.K. Das, D. Nongsiang, On the genus of the commuting graphs of finite non-abelian groups, Int. Electron. J. Algebra 19, 91-109, 2016.

[12] S. Dolfi, R.M. Guralnick, M. Herzog and C.E. Praeger, A new solvability criterion for finite groups, J. London Math. Soc. 85 (2), 269-281, 2012.

[13] J. Dutta and R.K. Nath, Spectrum of commuting graphs of some classes of finite groups, Matematika, 33 (1), 87-95, 2017.

[14] J. Dutta and R.K. Nath, Finite groups whose commuting graphs integral, Mat. Vesnik, 69 (3), 226-230, 2017.

[15] J. Dutta and R.K. Nath, Laplacian and signless Laplacian spectrum of commuting graphs of finite groups, Khayyam J. Math. 4 (1), 77-87, 2018.

[16] P. Dutta, J. Dutta and R.K. Nath, Laplacian spectrum of non-commuting graphs of finite groups, Indian J. Pure Appl. Math. 49 (2), 205-216, 2018.

[17] H.H. Glover, J.P. Huneke and C.S. Wang, 103 graphs that are irreducible for the projective plane, J. Combin. Theory Ser. B 27 (3), 332-370, 1978.

[18] R. Guralnick, B. Kunyavskii, E. Plotkin and A. Shalev, Thompson-like characterizations of the solvable radical, J. Algebra, 300 (1), 363-375, 2006.

[19] R.M. Guralnick and G.R. Robinson, On the commuting probability in finite groups, J. Algebra, 300 (2), 509-528, 2006.

[20] R. Guralnick and J. Wilson, The probability of generating a finite soluble group, Proc. London Math. Soc. 81 (3), 405-427, 2000. 
[21] D. Hai-Reuven, Non-solvable graph of a finite group and solvabilizers, arXiv:1307.2924v1, 2013.

[22] R.K. Nath and A.K. Das, On a lower bound of commutativity degree, Rend. Circ. Math. Palermo, 59 (1), 137-141, 2010.

[23] B.H. Neumann, A problem of Paul Erdös on groups, J. Aust. Math. Soc. (Ser. A), 21 (4), 467-472, 1976.

[24] D. Nongsiang, Double-Toroidal and Triple-Toroidal Commuting and Nilpotent Graph, Communicated.

[25] D. Nongsiang and P.K. Saikia, On the non-nilpotent graphs of a group, Int. Electron. J. Algebra, 22, 78-96, 2017.

[26] A.A. Talebi, On the non-commuting graphs of group $D_{2 n}$, Int. J. Algebra, 2 (20), 957-961, 2008.

[27] D.B. West, Introduction to Graph Theory (Second Edition), PHI Learning Private Limited, New Delhi, 2009.

[28] A.T. White, Graphs, Groups and Surfaces, North-Holland Mathematics Studies, no. 8., American Elsevier Publishing Co., Inc., New York, 1973.

[29] The GAP Group, GAP - Groups, Algorithms, and Programming, Version 4.6.4, 2013 (http://www.gap-system.org). 